\title{
Quality of Service Aware Multi-Hop Relay Networks for Green Radio Communication
}

\author{
M. Arthi*, P. Arulmozhivarman and K. Vinoth Babu \\ School of Electronics Engineering, VIT University, Vellore, Tamil Nadu, India \\ *Corresponding Author: arthimdas@gmail.com
}

Received 24 June 2015; Accepted 29 September 2015;

Publication 15 October 2015

\begin{abstract}
In recent years, issues on greenhouse pollution and power consumption related to the operation of information communication technology (ICT) devices have triggered a huge amount of research work towards green radio communication. Many of the power efficient solutions will introduce cost performance trade-offs. The energy efficient solution which also takes care of the quality requests of the mobile customer is really required for the $5 \mathrm{G}$ based green radio communication. It is identified that enhanced nodeBs (eNB) are power inefficient and not suited for many deployment scenarios. Multihop relay (MHR) network is the suitable alternate for eNB especially in the coverage holes and cell edges. But MHR network suffers by various quality issues like site planning and path selection. Many of the site planning and path selection schemes will fail under network imbalance conditions. In this work, we have proposed a hybrid throughput oriented (TO) - load aware spectral efficient routing (LASER) scheme which offers high performance even under network load imbalance conditions. The simulation results proved that the proposed scheme is more suited for future green radio communication.
\end{abstract}

Keywords: Green radio, LASER, path selection, site planning, TO scheme.

Journal of Green Engineering, Vol. 5, 85-106.

doi: 10.13052/jge1904-4720.521

(C) 2016 River Publishers. All rights reserved. 


\section{Introduction}

In recent years, green radio communication has become one of the hot research topics among the network operators, manufacturers and researchers [1]. In general, the term green technology is dedicated for the efforts to reduce the emissions of greenhouse gases from the industry. For telecommunication operators, the objectives of green approaches are to gain extra commercial benefits by reducing operating expenses related to energy cost. 5G wireless design is enormously propelled by energy efficiency perspectives. Green communication has pulled in a considerable measure of consideration because of rising electricity cost of general network operations and its unfriendly impact on environment due to $\mathrm{CO}_{2}$ emissions [2].

It has been noticed that the present wireless networks are not power efficient especially the eNBs $[1,2]$. The increase in the number of mobile users and the corresponding data rate demands, will increase the overall power consumption of the ICT industries. It is also noticed that $4 \%$ of the global $\mathrm{CO}_{2}$ emissions are from ICT industries. The cellular networks alone contribute $12 \%$ of the ICT $\mathrm{CO}_{2}$ emissions. In a cellular communication networks, almost $80 \%$ of the power is consumed by eNB sites. The power consumption of the eNB depends on the radio power transmitted, power consumed by digital signal processing transceivers, cooling units and the power back up units. The power consumed by the cooling units and back up units is more than $55 \%$ of the power consumed by the eNB. Present days, the number of eNBs has reached more than 4 million. The amount of $\mathrm{CO}_{2}$ emissions from eNB sites alone reached 78 million tons (approximately equal to the emissions from 15 million cars). It is anticipated that greenhouse gases emission from ICT devices will double or even triple in 2020. Manner et al. proved that in order to offer coverage similar to $2 \mathrm{G}$ network, Long term evolution (LTE) has to consume about 60 times amount of energy [3]. The power consumed and the amount of $\mathrm{CO}_{2}$ emissions by various components of cellular network is displayed in Table 1.

Many researchers are working on green communication to reduce the energy bills for telecomm service providers. In literature, there exist five different approaches to minimize the energy cost [5]. They are

1. Improving energy efficiency of hardware components.

2. Turning off the hardware components selectively.

3. Optimizing the energy efficiency of radio transmission.

4. Adopting renewable energy sources.

5. Planning and deploying small cells. 
Table 1 Comparison of power consumption and $\mathrm{CO}_{2}$ emissions by various network components [4]

\begin{tabular}{lll}
\hline Components & Power Consumption & $\mathrm{CO}_{2}$ Emissions $\left(\mathrm{CO}_{2} / \mathrm{a}\right)$ \\
\hline User Equipment (UE) & $0.1 \mathrm{~W}$ & $\sim 1 \mathrm{Mt}$ \\
eNB & $1 \mathrm{~kW}$ & $\sim 30 \mathrm{Mt}$ \\
$\begin{array}{l}\text { Network control (radio } \\
\text { network controller, base }\end{array}$ & $1 \mathrm{~kW}$ & $<0.5 \mathrm{Mt}$ \\
$\begin{array}{l}\text { station controller) } \\
\begin{array}{l}\text { Core and servers (mobile } \\
\text { switching centre, AAA }\end{array}\end{array}$ & $10 \mathrm{~kW}$ & \\
server, IP core) & & $\sim 7 \mathrm{Mt}$ \\
\hline
\end{tabular}

The first approach is energy efficient design of hardware components like power amplifier [6]. In a macro eNB, the input power is approximately $1500 \mathrm{~W}$. The air conditioning, signal processing and $\mathrm{AC}$ rectifier circuit takes powers of $200 \mathrm{~W}, 150 \mathrm{~W}$ and $150 \mathrm{~W}$ respectively. The remaining $65 \%$ of the input power (i.e. $1000 \mathrm{~W}$ ) alone given to the power amplifier. Based on the efficiency of the power amplifier much power is dissipated as heat. For example, $12 \%$ efficient power amplifier output will be $120 \mathrm{~W}$. The power amplifier output is given as the input to the antenna through a co-axial feeder. This operation introduces $50 \%$ loss. Thus almost $80 \%$ of the input power is dissipated as heat and the useful output power is only around the range $5 \%$ to $20 \%$. The power amplifier module with $35 \%$ of power efficiency for a small cell wideband code division multiple access (WCDMA) or LTE eNB costs around \$75 which covers a radius less than $2 \mathrm{~km}$ [7]. Increasing the power efficiency or coverage will also increase the implementation cost.

It has been identified that in LTE networks, $60 \%$ of power consumption is scaled with the traffic load [8]. As per European Telecommunication standards Institute (ETSI) definition, low load eNB requires $10 \%$ of radio frequency (RF) power. Medium load and busy load eNBs require $30 \%$ and $50 \%$ RF powers respectively. Thus in the second approach, eNB or some of the hardware components are put in the sleep mode during non-peak traffic hours. This may reduce unnecessary power consumptions like air conditioning etc. But this sleep mode approach may negatively impact on quality of service (QoS). It may decrease the capacity.

In the third approach, transmission energy is minimized by using the techniques like multi input multi output (MIMO), cognitive radio, energy efficient scheduling, channel coding etc [5, 9]. But again these approaches may increase the computational complexity. In the fourth approach instead of using the conventional energy resources like hydro carbon which produces 
greenhouse gases, it is suggested to use renewable resources like hydro, wind and solar powers [10]. The network operators in Bangladesh, Nigeria are already using solar operated eNBs $[11,12]$. Due to poor road and unsafe conditions, delivering the traditional energy resources like diesel is not always guaranteed. But again purchasing, replacing and installing new equipment's (including man power, transportation, and disruption of normal operation) may increase the cost.

The fifth approach is to introduce small cell networks like micro cell, pico cell or femto cell instead of macro eNBs [13]. The deployment cost and power consumption of small cells are much lower when compared to macro eNBs. The transmission power and deployment cost requirements of various LTE based eNBs are listed in Table 2. These small cells bring additional radio interferences when compared to the conventional homogeneous macro eNBs. Deploying more number of small cells may reverse the trend of energy saving. It also requires more overhead during the transmission.

The green radio requirements should also satisfy the customer demands like increase in average throughput per user and decrease in cost per bit [14]. The additive white Gaussian noise (AWGN), interference and shadowing effects will degrade the received signal quality and coverage [15]. The degradations can be overcome by deploying more number of eNB in the geographic area. Increase in the number of eNBs in a given geographic area, will increase the network cost, power consumption and interference. Thus to ensure green radio communication, the deployment of eNB should be minimized as much as possible without sacrificing the QoS.

MHR network is one of the suitable solutions to achieve energy efficiency. Long term evolution-advanced (LTE-A) Rel-10 and IEEE 802.16j standards have introduced the concept of MHR networks, where the Relay Nodes (RN) are used to improve the network performance along with the eNBs [16]. The transmission power and cost of the RNs are much smaller than eNB. The introduction of RNs may shorten the distance between the communicating nodes. This in turn reduces the transmission power required. RNs deployment is very useful in the areas where the eNB backhaul solutions

Table 2 Comparison of LTE eNBs [13]

\begin{tabular}{llr}
\hline eNB & Transmission Power & \multicolumn{1}{l}{ Cost } \\
\hline Macro & $20 \mathrm{~W}-160 \mathrm{~W}$ & 397,800 Euros \\
Micro & $2 \mathrm{~W}-20 \mathrm{~W}$ & 42,200 Euros \\
Pico & $250 \mathrm{~mW}-2 \mathrm{~W}$ & 12,400 Euros \\
\hline
\end{tabular}


(fibers, microwave links etc.) are unavailable and very expensive [17]. RNs can also be deployed in the areas where site acquisition for eNB deployment is very difficult. Since the deployment and removal can be done faster than the eNB, RNs are more preferable for temporary deployments than eNB. Deployment of RNs in the coverage holes will improve the received signal quality of surrounding UE. This in turn increases the total system capacity without much increasing the capital and operational expenses of the network operator. Because of the more beneficial nature and less power consumption, RNs are more likely to be deployed in the geographic area than the eNBs. This turns to be one of the best solutions for future green radio communication. But there exist some major issues like site planning and $\mathrm{RN}$ selection in MHR networks [16]. Until these issues addressed, MHR networks may not satisfy the customer demands in terms of throughput and cost. The unsatisfied customer will move to the competing operator.

Site planning and deployment of RNs in appropriate locations may reduce the total power required to serve the UEs [18]. Appropriate site planning will increase the system throughput and coverage. There exist several studies for eNB and RN site planning in literature [18-25]. Y. Yu et al. proposed a cost function based eNB and RN site planning scheme for IEEE 802.16j networks [19]. S-J. Kim et al. have proposed a cost effective coverage extension solution for mobile worldwide interoperability for microwave access (WiMAX) system [20]. The proposed solution analyzes the total deployment cost first and then determines the optimum number of eNBs and RNs based on the traffic demands. D. Yang et al. proposed a RN placement algorithm which minimizes the number of RNs to be deployed, using cooperative communication scheme and satisfies all the data rate requirements of the UEs [21]. H-C. $\mathrm{Lu}$ et al. proposed a heuristic scheme which determines the number of $\mathrm{RN}$ deployment locations for a given UE distribution and deployment budget [22]. H-C. Lu \& W. Liao proposed a joint eNB and RN deployment scheme for IEEE 802.16j networks [23]. The proposed scheme maximizes the system throughput by ensuring the total deployment cost within the limits of maximum allowed deployment budget. This scheme suffers by network load balancing issues. J-Y. Chang and Y-S. Lin proposed a novel uniform clustering based eNB and RN deployment scheme [24]. This scheme works in two phases, namely eNB-RN selection phase and eNB-RN deployment phase. This scheme provides reasonable throughput and coverage by balancing the network load between different eNBs. All the above mentioned schemes are computationally complex and many of the schemes encourage more eNB deployment which is not supported in 
green radio communication. L-C. Wang et al. proposed a low complex RN deployment scheme for IEEE $802.16 \mathrm{j}$ networks to maximize the network throughput [25]. The authors used TO and signal strength oriented (SSO) RN deployment schemes in their work. The results showed that the TO schemes offer more throughput than the SSO schemes. Thus in our proposed work, we adopt TO based RN deployment scheme to maximize the network throughput.

Once RNs are deployed, the performance improvements will not only depend on the site planning [21]. There exist a trade-off between system throughput and fairness. To offer high quality services to every user, appropriate set of RNs selection is important. There exist two different scenarios of MHR networks where RN selection is important. In the first scenario, an UE may be in the coverage area of both eNB and RN. In the second scenario, an UE may be covered by multiple RNs. But each UE should get the service from only one node. Thus, the path selection is an important issue in MHR networks. Improper path selection may lead to loss in system throughput and increase in the signal transmission delay [26, 27]. In literature, there exist many path selection schemes. Many of the powerful schemes like radio resource utilization index (RRUI), are also not addressed the link overloading issue. S-S. Wang et al. proposed a LASER path selection scheme which uses link cost as a metric for path selection [28]. This scheme offers better performance under link overloading conditions. Thus, in the proposed work, we adopt LASER based path selection scheme to address the link overloading issue.

The rest of the script is organized in the following order. The system model and the novel TO-LASER schemes are described in Sections 2 and 3 respectively. The simulation results are discussed in Section 4 and the paper is concluded in Section 5.

\section{System Model}

MHR network consists of three major nodes namely eNB, RN and UE [29]. LTE-A and IEEE 802.16j standards allow two different RN deployment scenarios [16]. In the first scenario, the RNs are deployed within the coverage area of eNB. These RNs are transparent RNs. This type of deployment will solve the coverage problems of the UEs especially in the coverage holes like indoor, underground etc. In the second scenario, RNs are deployed near the cell edge to extend the coverage. Such RNs are called non transparent RNs. In this work, site planning and RNs selection is done for both transparent and non-transparent RNs. All eNBs are connected to the mobile switching centre 
(MSC) which is acting as the gateway between wired and wireless networks. A typical MHR network is shown in Figure 1.

Based on the mobility, there exist three different types of RNs namely fixed RN (FRN), Nomadic RN (NRN) and Mobile RN (MRN). When there is a slow and group mobility, NRNs and MRNs are preferred respectively. NRNs are deployed in slow moving vehicles. MRNs are deployed in systems where there exist medium to high mobility like public transportation systems. Since the mobility of NRN is less, it can support certain number of users for certain amount of time without requiring more number of handoffs. Similarly, the group of users travelling in a train or bus will be getting the service from the MRN deployed in that transportation system. The connected users will be getting the service from the same MRN for most of the time. But the proposed work is limited to FRN. Identifying different types of RNs for different sites is out of scope for this work. The site planning for RNs should guarantee two features. The first one is, there should be a line of sight (LOS) from all RNs to eNB and the second one is there should be a non-line of sight (NLOS) from a RN to other interfering RNs. It is also assumed that eNB is deployed in the optimum location. Using the TO based site planning rule, the FRNs should be placed in the optimum distance from the eNB where the system throughput

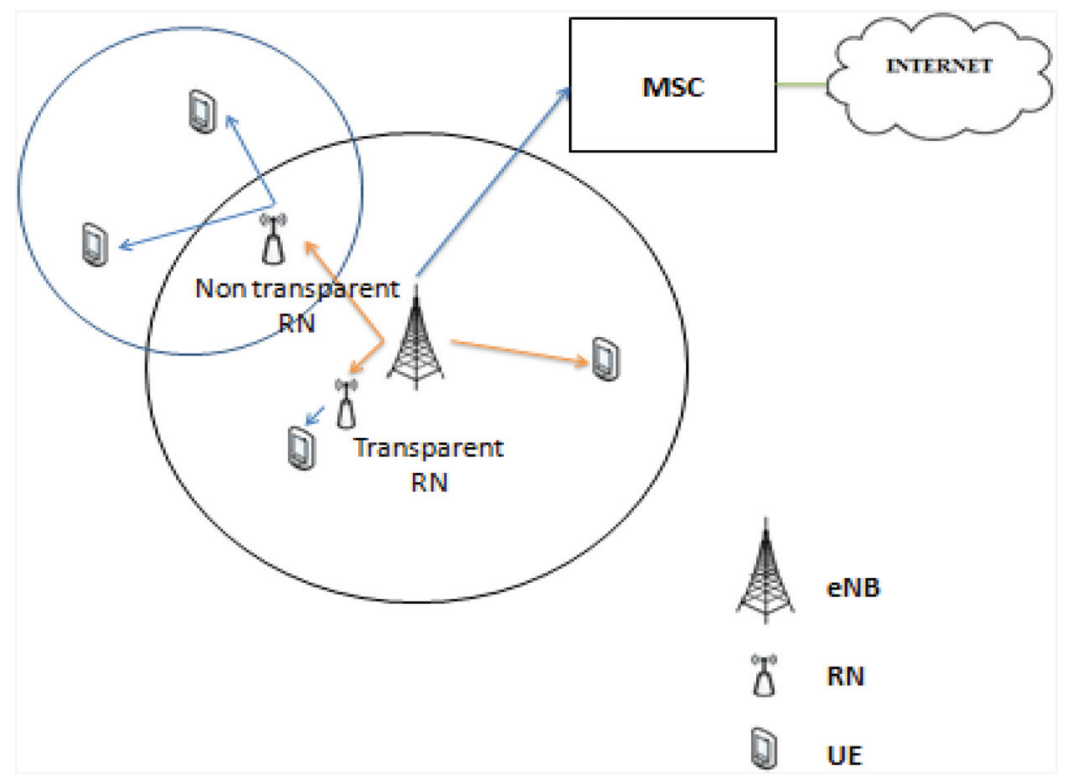

Figure 1 MHR network architecture. 
is maximum. At the end, the objective is to maximize the coverage ratio i.e each UE within the given geographic area must be connected to a eNB or RN or both.

For simple analysis, a free space propagation model is used to measure the received signal to noise ratio (SNR). The received SNR is given by [24],

$$
S N R(d B)=10 \log _{10}\left(\frac{\mathrm{P}_{R}}{\sigma_{N}^{2}}\right)
$$

where $\sigma_{N}^{2}$ is the noise variance and $\mathrm{P}_{R}$ is the received signal power which is given by

$$
P_{R}=P_{T}\left(\frac{c}{4 \pi f_{c} d}\right)^{2}
$$

where $P_{T}$ is the transmitted signal power, $f_{c}$ is the carrier frequency, $c$ is the velocity of light and $d$ is the distance between any two communicating nodes. The distance, received SNR and the corresponding burst profile is listed in Table 3.

The two phase transmission of the MHR network may reduce the system capacity [25]. It may also lead to unnecessary delay in transmission which is not desired in time bounded applications. To maximize the capacity and to minimize the transmission delay, the eNB has to decide for an indirect transmission, only when it is needed. This problem is more severe when the UEs are connected by both eNB and RN or by multiple RNs. Based on the TO rule, the indirect transmission is preferred as long as,

$$
R_{e N B-R N-U E}>R_{e N B-U E}
$$

Table 3 Modulation and coding scheme (MCS) for link adaptation [24]

\begin{tabular}{llllcc}
\hline $\begin{array}{l}\text { Burst } \\
\text { Profile }\end{array}$ & $\begin{array}{l}\text { Coding } \\
\text { Rate }\end{array}$ & Modulation & $\begin{array}{l}\text { Distance } \\
\text { between the } \\
\text { Nodes }(\mathrm{km})\end{array}$ & SNR $(\mathrm{dB})$ & $\begin{array}{l}\text { Data Rate } \\
(\mathrm{Mbps})\end{array}$ \\
\hline 1 & $1 / 2$ & BPSK & 3.20 & 3.00 & 1.269 \\
2 & $1 / 2$ & QPSK & 2.70 & 6.00 & 2.538 \\
3 & $3 / 4$ & QPSK & 2.50 & 8.50 & 3.816 \\
4 & $1 / 2$ & 16-QAM & 1.90 & 11.50 & 5.085 \\
5 & $3 / 4$ & 16-QAM & 1.70 & 15.00 & 7.623 \\
6 & $2 / 3$ & 64-QAM & 1.30 & 19.00 & 10.161 \\
7 & $3 / 4$ & 64-QAM & 1.20 & 21.00 & 11.439 \\
\hline
\end{tabular}


where $R_{e N B-R N-U E}$ is the data transmission rate of indirect transmission and $R_{e N B-U E}$ is the direct data transmission rate.

The data transmission rate of indirect transmission is given by,

$$
\begin{aligned}
R_{e N B-R N-U E} & =\frac{D}{t_{e N B-R N-U E}}=\frac{D}{\frac{D}{R_{e N B-R N}}+\frac{D}{R_{R N-U E}}} \\
& =\left(\frac{1}{R_{e N B-R N}}+\frac{1}{R_{R N-U E}}\right)^{-1}
\end{aligned}
$$

where $D$ is the packet size and $t_{e N B-R N-U E}$ is the time taken for indirect transmission. Based on the TO scheme, the data transmission rate for an UE is

$$
R=\operatorname{Max}\left(R_{e N B-R N-U E}, R_{e N B-U E}\right)
$$

The average system capacity can be obtained using,

$$
C=\frac{\sum_{k=1}^{M} R(k)}{M}
$$

where $M$ is the total number of UEs in the given geographic area and $R(k)$ is the data transmission rate of the $k^{\text {th }} \mathrm{UE}$.

The concept of TO selection rule is illustrated in Figure 2. Based on (5), there are chances that multiple UEs may choose a single RN for service. The functional and capacity limitations of RNs may introduce link overloading issue [28]. This will reduce the net system throughput when it is

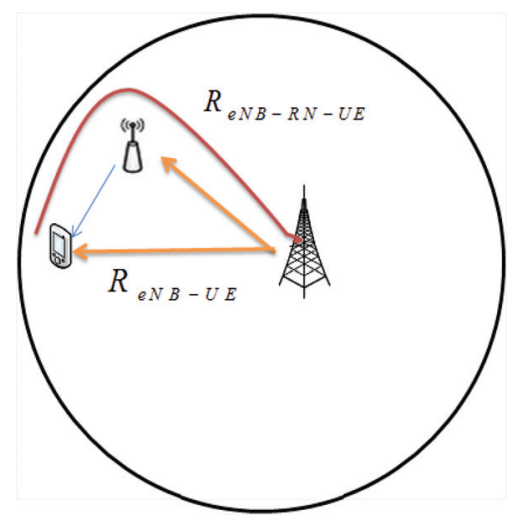

Figure 2 The concept of TO selection rule. 


\section{M. Arthi et al.}

left uncared. The TO selection rule will not address the link overloading issue. A LASER based RN selections rule will address the link overloading issue when compared to other $\mathrm{RN}$ selection rules. The concept of link overloading in MHR network is shown in Figure 3.

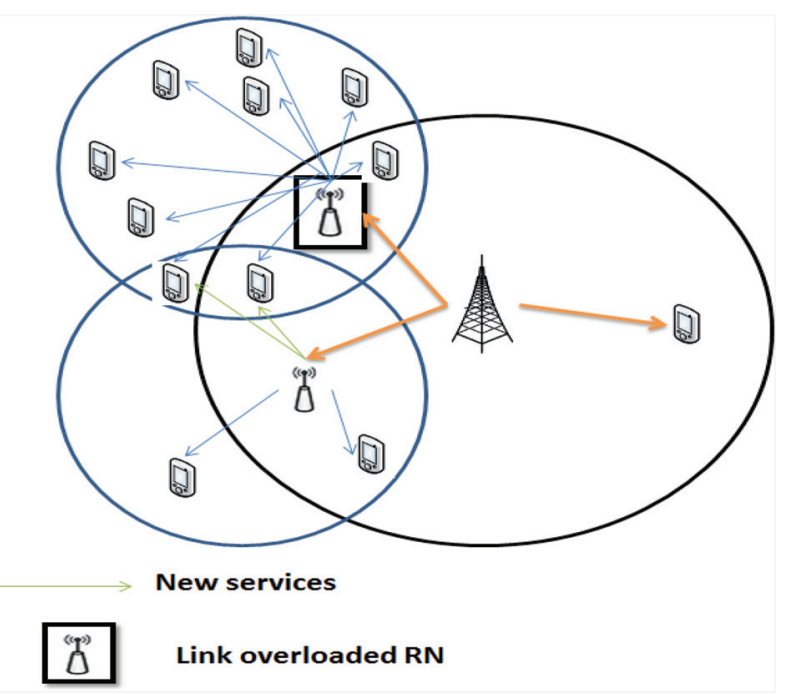

Figure 3 Link overloading.

Table 4 Notations list

\begin{tabular}{ll}
\hline Notations & Descriptions \\
\hline$C(i)$ & network capacity at $i^{t h}$ distance \\
$C_{R N}$ & capacity of the RN \\
$X$ & coordinates of eNB \\
$Y_{k}$ & coordinates of the $k^{t h} \mathrm{UE}$ \\
$Z_{j}^{i}$ & coordinates of $j^{t h} \mathrm{RN}$ under $i^{t h}$ distance \\
$d_{i}$ & set of $i^{t h}$ possible distance for RNs deployment \\
$d_{\text {max }}$ & maximum possible eNB-RN separation distance \\
$d_{o p t}$ & optimum distance for RNs deployment with maximum network \\
& capacity \\
$e 1$ & distance between eNB to $j^{t h} \mathrm{RN}$ for $i^{t h}$ distance \\
$e 2$ & distance between $j^{t h} \mathrm{RN}$ and $k^{t h} \mathrm{UE}$ \\
$e 3$ & distance between eNB and $k^{t h} \mathrm{UE}$ \\
$l$ & number of possible eNB RN distances \\
$m$ & number of RNs in the geographic area \\
$z_{j}$ & $j^{t h} \mathrm{RN}$ \\
\hline
\end{tabular}




\section{The Proposed TO-LASER Scheme}

In the proposed scheme, along the vertices of eNB, the set of possible equal distances is taken for RNs deployment. The algorithm initially calculates the distance between eNB and RN. It also calculates the distance between each UE with all the RNs in the geographic area. Based on this, each UE identifies the nearby RN. The direct path distance i.e the distance between eNB and UE

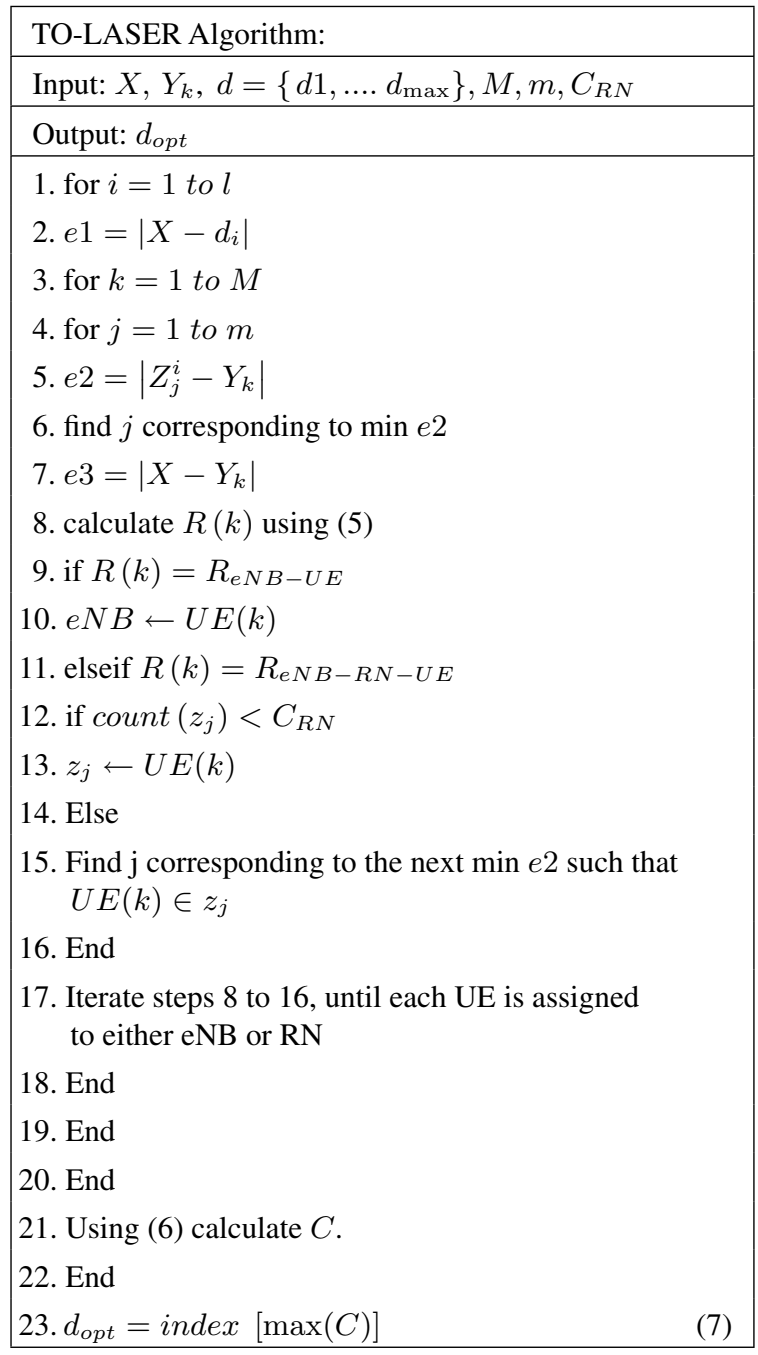


is also calculated. Based on (5), the necessity for indirect communication is tested. To check the link overloading condition, the capacity of the nearby $\mathrm{RN}$ is checked. If it has less number of UEs compared to its capacity, then the particular UE is assigned to the corresponding RN. Otherwise the next nearby $\mathrm{RN}$ is checked for its capacity. This process is repeated, until each UE identifies a RN without link overloading. After assigning all the UEs to either eNB or to any of the nearby RNs, the overall network capacity can be obtained using (6). This process is repeated for all possible set of distances taken for the RNs deployment. Finally the distance for which the maximum network capacity is attained is assumed to be the optimum location for RNs deployment under link overloading condition.

\section{Simulation Results and Discussion}

In this section, a comparison of different path selection schemes in terms of fairness, spectral efficiency and average transmission power per $\mathrm{UE}$ is done. The schemes considered are no relaying, RRUI relaying, LASER relaying and joint TO-LASER relaying. The simulations are repeated for 100 different scenarios and the average results are displayed in this work. The following parameters and assumptions are considered for the simulations:

- The geographic region is assumed to be of $10 \mathrm{~km} \times 10 \mathrm{~km}$.

- The MHR network consists of three major nodes such as eNB, RN and UE.

- $\mathrm{RN}$ is considered to be FRN.

- It is assumed that eNB is located in an optimum location.

- eNB and RNs use Omnidirectional antennas.

- eNB and RNs are assumed to have a coverage radius of $3.2 \mathrm{~km}$ and $1.9 \mathrm{~km}$ respectively.

- 100 UEs are deployed randomly in the geographic region with nonuniform traffic demands.

- It is assumed that all the RNs are in the LOS with eNB.

- The RNs are placed with sufficiently far distance, so that the co-channel interference among them is minimum.

- The candidate locations of the RNs are assumed to be on the direction of the vertices of the hexagonal eNB.

- The path loss calculations are done based on the free space propagation model. 
- It is assumed that perfect channel state information (CSI) is available at the transmitter and receiver.

- No particular mobility model is considered in this work.

The average system throughput (Mbps) vs. eNB-RN separation distance is showed in Figure 4. The proposed TO-LASER scheme is executed to find the optimal distance for RNs deployment. Since there exist only direct communication in no relaying scheme, the average system throughput is constant irrespective of the eNB-RN separation distance. For the TO-LASER scheme, the average system throughput increases with the increase in eNB-RN separation distance up to $1.9 \mathrm{~km}$ and then it decreases. The system throughput is maximum at $1.9 \mathrm{~km}$. This is almost same for different UE distributions with different traffic demands. Thus the RNs are deployed at the distance of $1.9 \mathrm{~km}$ from the eNB. We assume hexagonal cell shape and the RNs are deployed on the directions of the vertices of the hexagonal. This is clear from Figure 5.

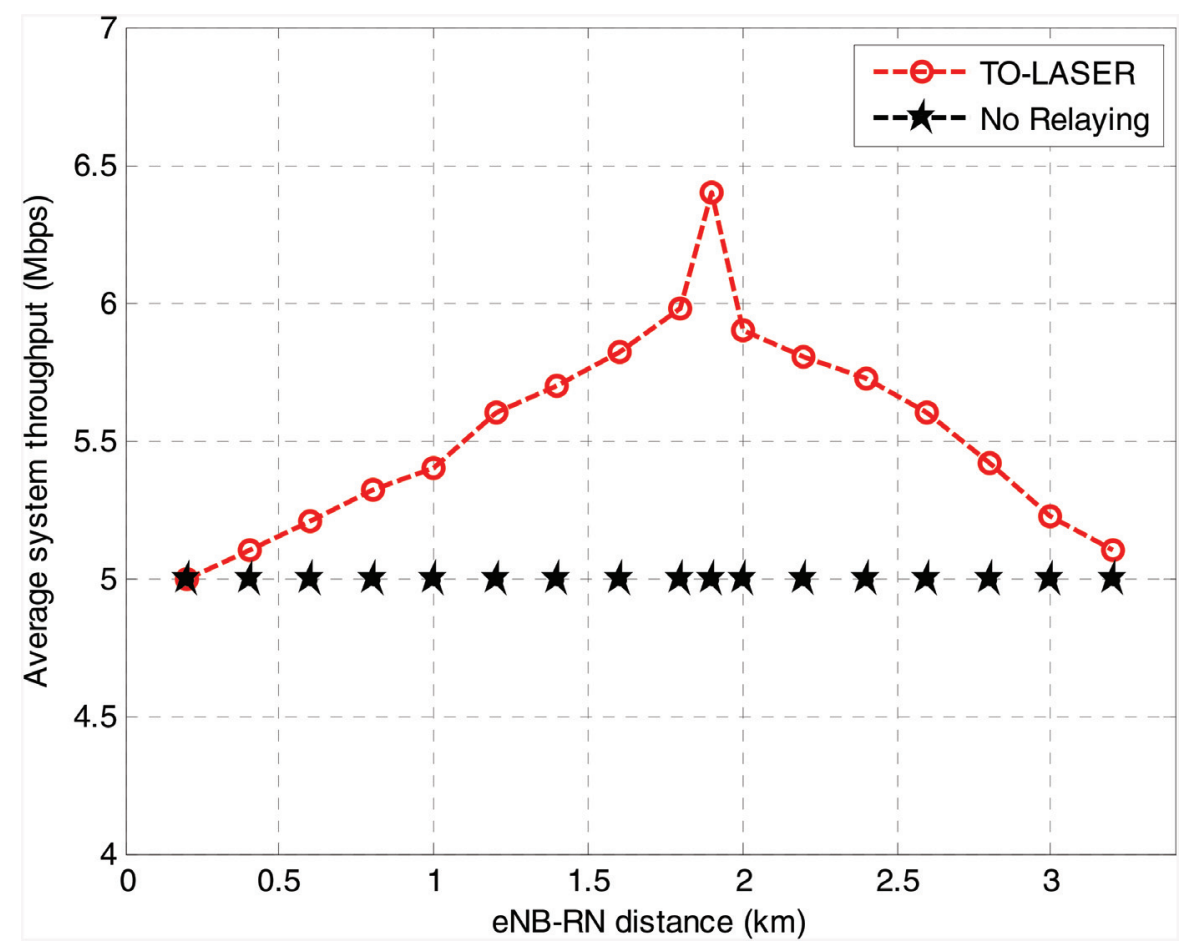

Figure 4 Optimal RN location identification using TO-LASER based selection rule. 


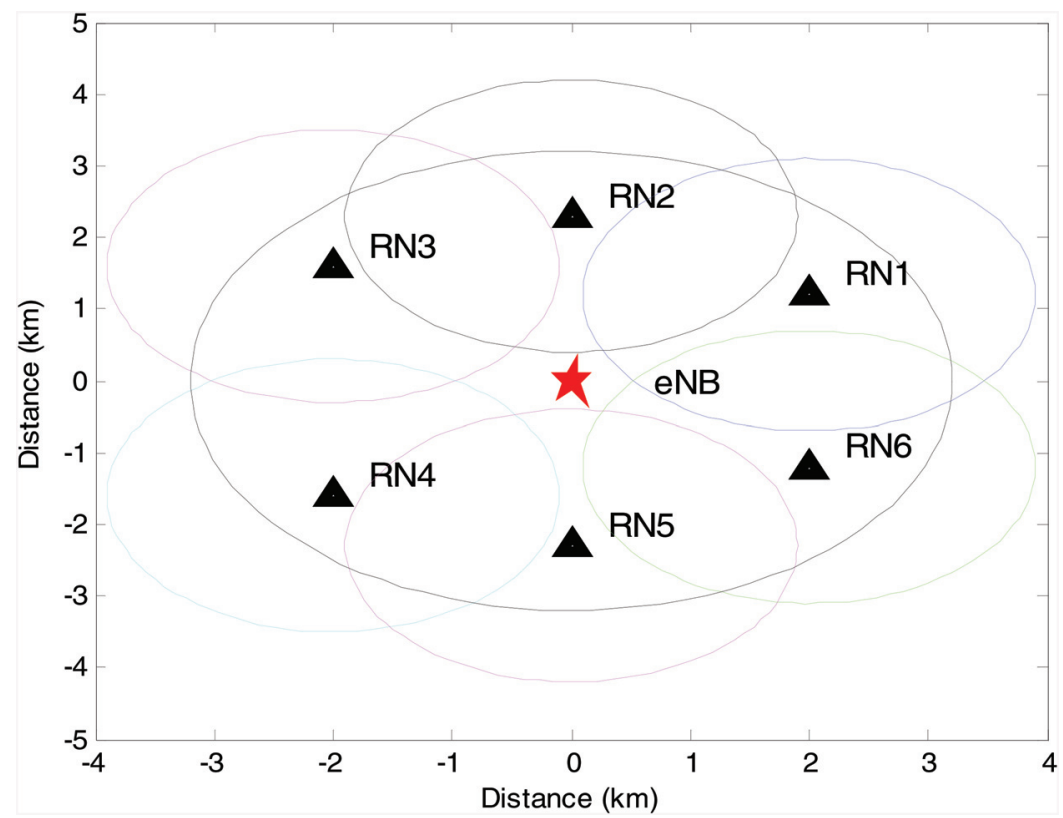

Figure 5 eNB and RNs locations after TO-LASER based site planning scheme.

For comparison, we introduce utilization termed $\rho$ which represents the congestion level of the traffic load in the system. This factor defines the proportion of the system resources used by the traffic in a system.

$$
\rho=\frac{\gamma}{\eta}
$$

where $\gamma$ and $\eta$ are the mean arrival rate and the mean service rate of a system. The value of utilization in the simulation ranges from 0.1 to 1.0 with a step of 0.1 .

Figure 6 shows the average spectral efficiency $(\mathrm{b} / \mathrm{s} / \mathrm{Hz})$ of various schemes under different utilization. The average spectral efficiencies attained by no relaying, RRUI relaying, LASER relaying and TO-LASER relaying schemes are $0.4758,0.8405,1.1324$ and 1.2574 respectively. The spectral efficiency performance of LASER relaying is far better than the other two schemes (no relaying and RRUI relaying) under link overloading conditions. The inclusion of TO based site planning along with the LASER based relaying, improves the average spectral efficiency to $9.9729 \%$ than LASER relaying scheme. 


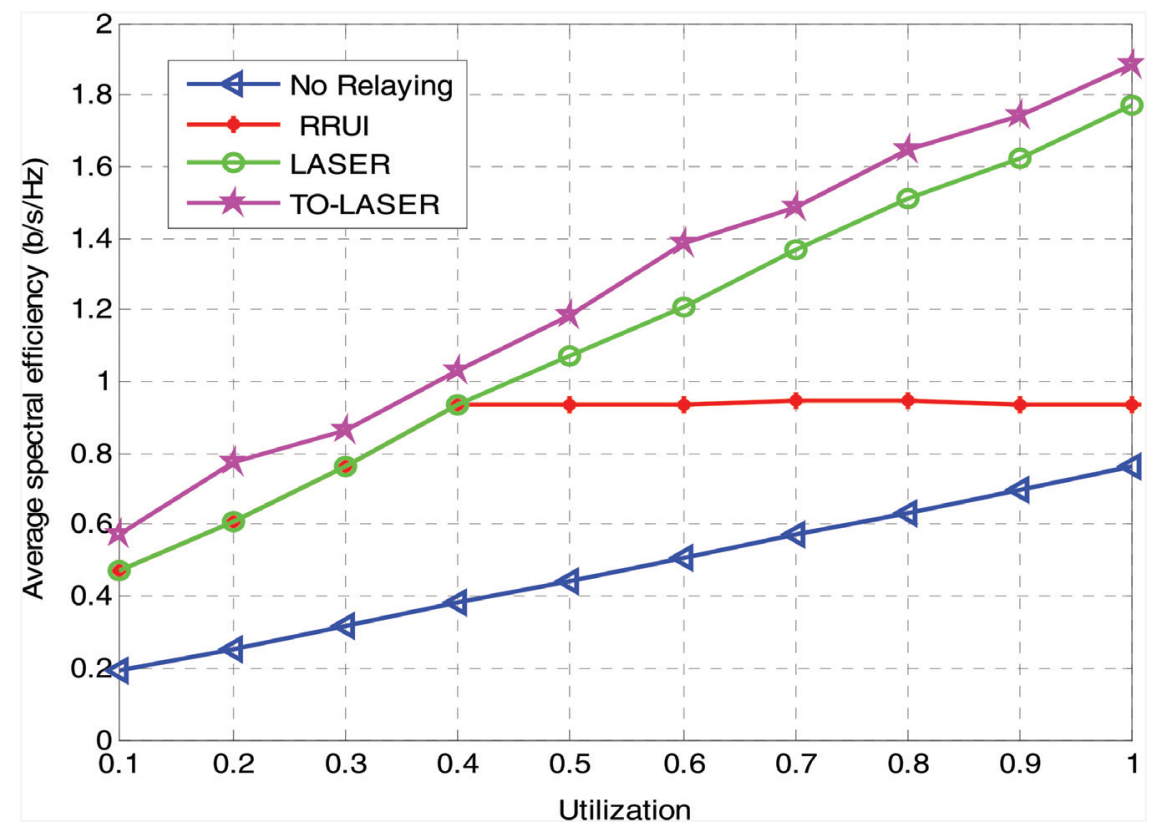

Figure 6 Average spectral efficiency (b/s/Hz) comparison for various path selection schemes.

Figure 7 indicates the fairness of various schemes under different utilization. Fairness determines whether the network under different utilization is receiving a fair share of system resources. It is given by,

$$
F=\frac{\left(\sum_{k=1}^{M} R(k)\right)^{2}}{M \sum_{k=1}^{M}(R(k))^{2}}
$$

It shows that the fairness of TO-LASER and LASER schemes remain same for different utilization values. It shows that all the UEs under the eNB coverage area are receiving a fair share of resources. For RRUI relaying, the fairness begins to drop gradually when it reaches a utilization of 0.4 . This is due to the limited link capacity. Even though the no relaying scheme attains maximum fairness under different utilization, it is not preferred due to low spectral efficiency.

The total power consumption is the addition of transmission power and power consumed by the hardware equipment. The downlink data transmission rate of a $\mathrm{k}^{\mathrm{th}} \mathrm{UE}$ is given by, 


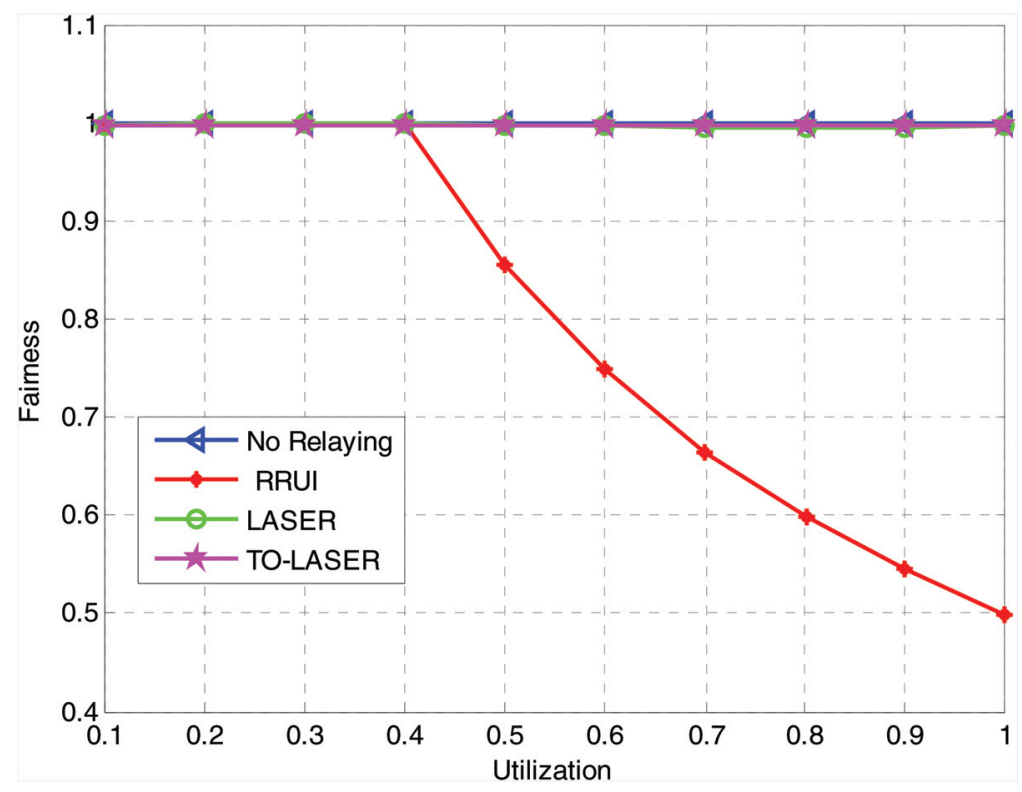

Figure 7 Fairness comparison for various path selection schemes.

$R_{e N B-U E(k)}=B_{e N B-U E(k)} \log _{2}\left(1+\frac{P_{e N B-U E(k)}}{\sigma_{N}^{2}}\left(\frac{c}{4 \pi f_{c} d_{e N B-U E(k)}}\right)^{2}\right)$

where $B_{e N B-U E(k)}$ is the bandwidth allocated for downlink transmission between eNB and $k^{t h} \mathrm{UE}$ and $d_{e N B-U E(k)}$ is the distance between eNB and $k^{t h}$ UE. The power required for downlink transmission between eNB and $k^{t h}$ UE is given by,

$$
P_{e N B-U E(k)}=\left(2^{\frac{R_{e N B-U E(k)}}{B_{e N B-U E(k)}}}-1\right)\left(\frac{4 \pi f_{c} d_{e N B-U E(k)}}{c}\right)^{2} \sigma_{N}^{2}
$$

The introduction of RNs will lessen the transmission distance between the communicating nodes. As per (11), the transmission power is directly proportional to the distance. Thus the properly deployed RNs will reduce the total transmission power for achieving a desired fixed rate of transmission. 
The total transmission power for no relay scheme is calculated using,

$$
P_{T}=\sum_{k=1}^{M} P_{e N B-U E(k)}
$$

The total transmission power for MHR is calculated using,

$$
P_{T}=\sum_{k_{1}=1}^{M_{1}} P_{e N B-U E\left(k_{1}\right)}+\sum_{k_{2}=1}^{M_{2}} P_{e N B-R N-U E\left(k_{2}\right)}
$$

where $\mathrm{M}_{1}$ and $\mathrm{M}_{2}$ are the number of UEs connected directly and indirectly with eNB. $P_{e N B-R N-U E\left(k_{2}\right)}$ is the transmission power required for an indirect transmission.

For 100 different UE distributions with utilization 0.5, the average transmission power per UE (in W) is shown in Figure 8. The graph is developed based on the assumption of average AWGN with power spectral density of $-174 \mathrm{dBm} / \mathrm{Hz}$ and $100 \mathrm{UEs}$. The average transmission power per UE for no relaying, RRUI, LASER, TO-LASER schemes are $-46.95 \mathrm{dBm},-50.41 \mathrm{dBm}$, $-51.22 \mathrm{dBm},-53.49 \mathrm{dBm}$ respectively. We need a minimum of four eNBs to cover the considered geographic area. The total amount of power required by

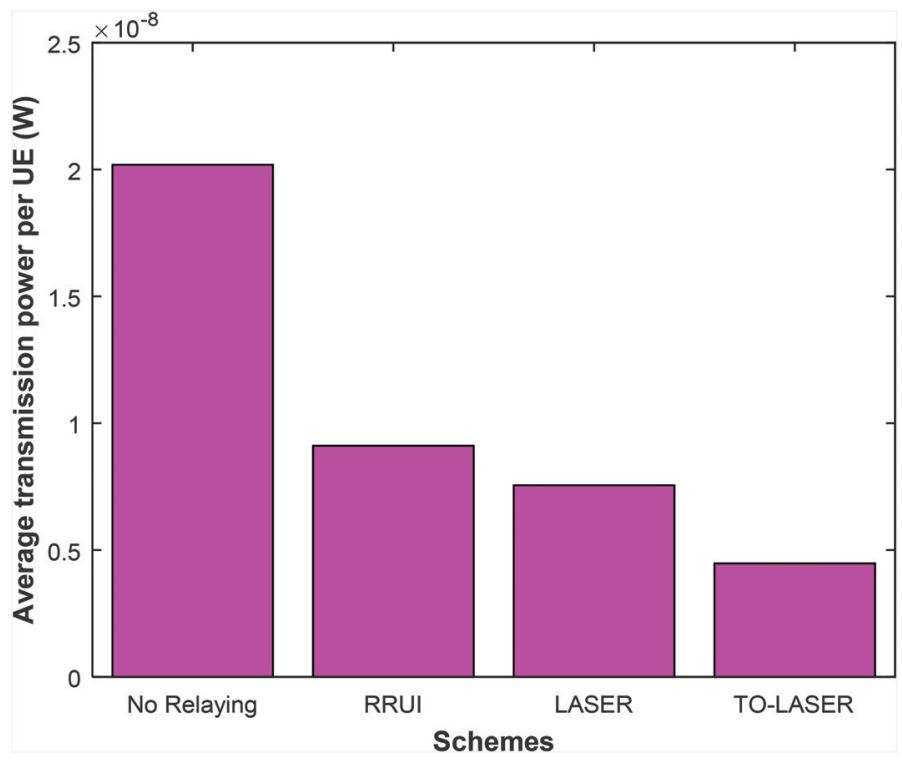

Figure 8 Average transmission power per UE (W) comparison for different schemes. 
the eNBs is approximately $6000 \mathrm{~W}$. When we go for TO-LASER scheme, we need one eNB and six RNs which consume the total power approximately of $2500 \mathrm{~W}$. This power consumption is pretty much same for other relay based schemes considered. Be that as it may, the other relay based schemes, require more transmission power than the TO-LASER scheme. Thus the total power required for TO-LASER is much lesser than the other schemes.

\section{Conclusions}

This paper has discussed the importance of RN based MHR networks for green radio communications. There exist some severe issues like site planning and path selection in MHR networks. In this work, a hybrid TO-LASER site planning scheme is proposed which offers high performance than the conventional LASER based scheme even at link overloading conditions. It shows a $9.9729 \%$ improvement in spectral efficiency over the conventional LASER scheme. The proposed scheme also offers better fairness in terms of resource allocation. The average transmission power per UE for TO-LASER scheme is $-53.49 \mathrm{dBm}$ which is much lower than the other considered schemes. All the relay based schemes require only $40 \%$ of the total power consumed by no relaying scheme. The proposed deployment and path selection concepts can be extended to femto relays. The real time impairments like channel estimation error and channel information feedback delay are not considered in this work. The path selection scheme used here is for downlink. The promising results pave path for further investigations on uplink path selection schemes under various channel impairments and co channel interference conditions.

\section{References}

[1] P. Serrano; A. de la Oliva; P. Patras; V. Mancuso; A. Banchs, Greening Wireless Communications: Status and Future Directions, Comput. Commun., 35:1651-1661 (2012).

[2] I. Ahmed; M. M. Butt; C. Psomas; A. Mohamed; I. Krikidis; M. Guizani, Survey on Energy Harvesting Wireless Communications: Challenges and Opportunities For Radio Resource Allocation. Comput. Netw., 88:234-248 (2015).

[3] J. Manner; M. Luoma; J. Ott; J. Hamalainen, Mobile networks unplugged. In Proceedings of the 1st International Conference on Energy-Efficient Computing and Networking, pp. 71-74 (2010). 
[4] A. T. Clark, Nokia Siemens Networks Environmentally Sustainable Business Good green business sense [Online]. Available at http:// www.ist emobility.org/WorkingGroups/Applications/Meeetings-Events/ 2010-05-19_Athens/AClark.pdf.

[5] J. Wu; Y. Zhang; M. Zukerman; E. Yung, Energy-efficient Base-stations Sleep-mode Techniques in Green Cellular Networks: A Survey, IEEE Commun. Surv. Tuts., 17(2):803-826 (2015).

[6] C. Han et al., Green Radio: Radio Techniques to Enable Energy-efficient Wireless Networks. IEEE Commun. Mag., 49(6):46-54 (2011).

[7] TriQuint, 'RF Power Amplifier Module: TGA2450-SM' [Online] Available at http://store.triquint.com/ProductDetail/TGA2450-SMTri Quint-Semiconductor-Inc/472292/.

[8] I. Ashraf; F. Boccardi; L. Ho, Sleep Mode Techniques for Small Cell Deployments. IEEE Commun. Mag., 49(8):72-79 (2011).

[9] D. Feng et al., A Survey of Energy-efficient Wireless Communications. IEEE Commun. Surv. Tuts., 15(1):167-178 (2013).

[10] Y.-K. Chia; S. Sun; R. Zhang, Energy Cooperation in Cellular Networks with Renewable Powered Base Stations, IEEE Trans. Wireless Commun. 13(12):6996-7010 (2014).

[11] Y. Chan, China's Huawei to Supply Solar-Powered Base Stations to Bangladesh. Business Green, London, UK [Online]. Available at http://www.businessgreen.com/bg/news/1802444/, chinas-huaweisupply-solar-powered-base-stations-bangladesh (2009).

[12] C. Okoye. Airtel Base Stations to be Solar Powered, Daily Times Nigeria, Lagos, Nigeria [Online]. Available at http://dailytimes.com.ng/article/ airtel-base-stations-be-solar-powered (2011).

[13] Y.-C. Wang; C.-A. Chuang, Efficient eNB Deployment Strategy for Heterogeneous Cells in 4G LTE Systems. Comput. Netw. 79: 297-312 (2015).

[14] 3GPP TR 36.806 V9.0.0 3rd Generation Partnership Project, Technical Specification Group Radio Access Network, Evolved Universal Terrestrial Radio Access (E-UTRA), Relay architectures for E-UTRA (LTE-Advanced) (Release 9), http://www.qtc.jp/3GPP/Specs/36806900.pdf, 2010-13.

[15] M. Arthi; P. Arulmozhivarman; K. VinothBabu; J. Jose Joy; E. Mariam George, Technical Challenges in Mobile Multi-hop Relay Networks. Int. J. Appl. Eng. Res., 10:26025-26036 (2015).

[16] M. Arthi; P. Arulmozhivarman; K. VinothBabu; G. Ramachandra Reddy; D. Barath, Techniques to Enhance the Quality of Service of Multi hop Relay Networks. Proc. Comput. Sci., 46:973-980 (2015). 
104 M. Arthi et al.

[17] I. F. Akyildiz; E. Chavarria-Reyes; D. M. Gutierrez-Estevez; R. Balakrishnan, LTE-Advanced and the Evolution to Beyond 4G (B4G) systems. Phys. Commun., 10:31-60 (2014).

[18] B.-J. Chang; Y.-H. Liang; S.-S. Su, Analyses of Relay Nodes Deployment in $4 \mathrm{~g}$ Wireless Mobile Multihop Relay Networks, Wireless PersCommun. doi: 10.1007/s11277-015-2443-x (2015).

[19] Y. Yu; S. Murphy; L. Murphy, Planning base station and relay station locations in IEEE 802.16j multi-hop relay networks. In Consumer communications and networking conference, pp. 922-926 (2008).

[20] S.-J. Kim; S.-Y. Kim; B.-B. Lee; S.-W. Ryu; H.-W. Lee; C.-H. Cho, Multi-hop Relay Based Coverage Extension in the IEEE802.16j Based Mobile WiMAX Systems. In Fourth international conference on networked computing and advanced information management, Vol. 1, pp. 516-522 (2008).

[21] D. Yang; X. Fang; G. Xue; J. Tang, Relay Station Placement for Cooperative Communications in WiMAX Networks. In Proceedings of the IEEE global telecommunications conference, (GLOBECOM) (2010).

[22] H.-C. Lu; W. Liao; F. Y-S. Liu, Relay Station Placement Strategy in IEEE 802.16j WiMAX Networks. IEEE Trans. Commun., 59:151-158 (2011).

[23] H.-C. Lu; W. Liao, Joint Base Station and Relay Station Placement for IEEE 802.16j Networks. In Proceedings of IEEE global telecommunications conference, (GLOBECOM) (2009).

[24] J.-Y. Chang; Y.-S. Lin, A Clustering Deployment Scheme for Base Stations and Relay Stations in Multi-Hop Relay Networks. Comput. Electric. Eng. 40(2):407-420 (2014).

[25] L.-C. Wang; W.-S. Su; J.-H. Huang; A. Chen C.-J. Chang, Optimal Relay Location in Multi-Hop Cellular Systems. In Proceedings of the IEEE wireless communications and networking conference (WCNC) (2008).

[26] S.-S. Wang; H.-C. Yin; Y.-H. Tsai; S.-T.Sheu, An Effective Path Selection Metric for IEEE 802.16-based Multi-hop Relay Networks. In Proceedings of the IEEE symposium on computers and communications, pp. 1051-1056 (2007).

[27] D. D. Couto; D. Aguayo; J. Bicket; R. Morris, A High-throughput Path Metric for Multi-hop Wireless Routing. In Proceedings of the ACM international conference on mobile computing and networking (MOBICOM), pp. 134-146 (2003). 
[28] S.-S. Wang, C.-Y. Lien, W.-H. Liao and K.-P. Shih. LASER, A Loadaware Spectral-efficient Routing metric for Path Selection in IEEE 802.16j Multi-hop Relay Networks. Comput. Electric. Eng., 38:953-962 (2012).

[29] D. Satishkumar; N. Nagarajan, Relay Technologies and Technical Issues in IEEE 802.16j Mobile Multi-hop Relay (MMR) Networks. J. Netw. Comput. Appl., 36:91-102 (2013).

\section{Biographies}

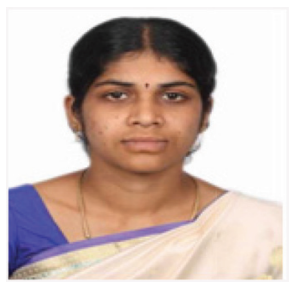

M. Arthi is currently working as a Research Associate in School of Electronics Engineering, VIT University, Vellore, Tamil Nadu, India. She has completed her B.E and M.E degrees from Anna University, Chennai, India in 2010 and 2012 respectively. Her area of interests includes Wireless networks and computer communication networks.

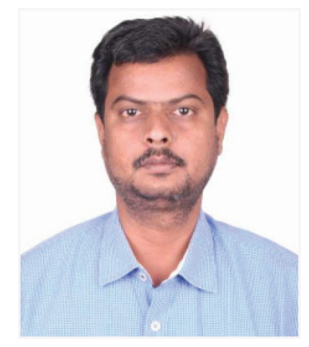

P. Arulmozhivarman is currently working as a Professor in School of Electronics Engineering, VIT University, Vellore, Tamil Nadu, India. He is the program chair for Electronics and Communication Engineering department, Division chair for Digital Signal Processing division and Assistant Dean for School of Electronics Engineering. He has completed his Ph.D degree from National Institute of Technology, Trichy in 2005. His area of interests includes Digital Signal Processing, Digital Image processing, neural networks and 
pattern recognition etc. He served as one of the editor in several international conference proceedings and he is a reviewer of International Journal of Computer Engineering Research and IEEE Photonics Technology letters. Dr. P. Arulmozhivarman is a member of IEEE signal processing society.

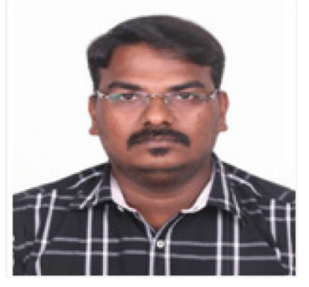

K. Vinoth Babu is currently working as an Associate Professor in School of Electronics Engineering, VIT University, Vellore, Tamil Nadu, India. He has completed his Ph.D and M.Tech degrees from VIT University, Vellore, India in 2014 and 2009 respectively and B.E (ECE) Degree from Anna University, Chennai, India in 2005. His area of interests includes Wireless Digital Communications and Signal Processing. 